\title{
A lateralidade e o desenvolvimento motor infantil de crianças quilombolas
}

\author{
The laterality and the child motor development of quilombola children \\ Lateralidad y desarrollo motor infantil de niños quilombolas
}

\author{
Alex Lima Pinto \\ ORCID: https://orcid.org/0000-0002-3987-7227 \\ Centro Universitário da Amazônia, Brasil \\ E-mail: alexunifisio@gmail.com \\ Karen Pereira Moreira Lima \\ ORCID: https://orcid.org/0000-0003-1120-1900 \\ Centro Universitário da Amazônia, Brasil \\ E-mail: karemmoreira@icloud.com \\ Assis Júnior Cardoso Pantoja \\ ORCID: https://orcid.org/0000-0002-8301-3135 \\ Universidade do Estado do Pará, Brasil \\ E-mail: assisfisio_jr@hotmail.com \\ Rafaela dos Santos Reis \\ ORCID: https://orcid.org/0000-0003-0127-0072 \\ Universidade Federal do Oeste do Pará, Brasil \\ E-mail: rafaelaibef@gmail.com \\ Maiara Silvana Salgado Batista \\ ORCID: https://orcid.org/0000-0003-2884-8794 \\ Universidade da Amazônia, Brasil \\ E-mail: maiarasilvana@hotmail.com \\ Iani Dias Lauer Leite \\ ORCID: https://orcid.org/0000-0001-9063-475X \\ Universidade Federal do Oeste do Pará, Brasil \\ E-mail: iani.leite@ufopa.edu.br
}

\begin{abstract}
Resumo
Objetivo: Analisar a lateralidade de crianças quilombolas e sua relação com o desenvolvimento motor. Metodologia: Trata-se de um estudo exploratório observacional, de caráter analítico, do tipo descritivo e transversal, com abordagem quantitativa e qualitativa, realizada na comunidade quilombola de Tiningu, Santarém-Pará. A amostra foi composta por 18 crianças de ambos os sexos com idade entre 7 e 11 anos. Como instrumento de coleta de dados foi utilizada a EDM proposta por Rosa Neto (2002). Os dados foram tabulados e analisados de acordo com a proposta de Rosa Neto (2002) e passaram por análise estatística descritiva simples no Microsoft Office Excel 2010 e BioEstat versão 5.0. Resultados e discussão: A faixa etária em maior quantidade se concentra nas idades de 10 a 11 anos. Os resultados de lateralidade mostram que a maioria das crianças $(72,22 \%)$ eram destras completas, $(27,78 \%)$, apresentaram lateralidade indefinida e nenhuma criança apresentou lateralidade sinistra ou cruzada. Conclusão: É importante analisar a lateralidade das crianças, pois a partir dessa habilidade, elas podem dominar seus movimentos e conhecer a si mesmas e o mundo ao seu redor. Sugere-se a realização de estudos similares, com amostras representativas, apontando dados que chamem a atenção para uma visão mais ampla da lateralidade em crianças, como prevenção e intervenção de prováveis distorções no processo de aprendizagem.
\end{abstract}

Palavras-chave: Desenvolvimento motor; Lateralidade; Crianças quilombolas.

\begin{abstract}
Objective: To analyze the laterality of quilombola children and its relationship with motor development. Methodology: This is an exploratory, observational, analytical, descriptive and cross-sectional study, with a quantitative and qualitative approach, carried out in the quilombola community of Tiningu, Santarém-Pará. The sample consisted of 18 children of both genders aged between 7 and 11 years. As a data collection instrument, an EDM proposed by Rosa Neto (2002) was used. Data were tabulated and according to the proposal of Rosa Neto (2002) and underwent simple descriptive statistical analysis in Microsoft Office Excele 2010 and BioEstat version 5.0. Results and discussion: The age group in greater quantity is concentrated in the ages of 10 to 11 years. The laterality results show that the majority of children $(72.22 \%)$ were complete right-handed, $(27.78 \%)$ loss of indefinite laterality and no children presented sinister or crossed laterality. Conclusion: It is important to analyze the laterality of children because from these skills, they can master their movements and know themselves and the world around them. Carrying out similar studies, with representatives, taking data that draw attention to a broader view of laterality in children, such as prevention and intervention of probable distortions in the learning process.
\end{abstract}

Keywords: Motor development; Laterality; Quilombola children. 


\begin{abstract}
Resumen
Objetivo: Analizar la lateralidad de los niños quilombolas y su relación con el desarrollo motor. Metodología: Se trata de un estudio exploratorio, observacional, analítico, descriptivo y transversal, con enfoque cuantitativo y cualitativo, realizado en la comunidad quilombola de Tiningu, Santarém-Pará.. La muestra estuvo formada por 18 niños de ambos sexos con edades comprendidas entre los 7 y los 11 años. Como instrumento de recolección de datos se utilizó el EDM propuesto por Rosa Neto (2002). Los datos se tabularon y analizaron según la propuesta de Rosa Neto (2002) y se sometieron a análisis estadístico descriptivo simple en Microsoft Office Excele 2010 y BioEstat versión 5.0. Resultados y discusión: El grupo de edad en mayor cantidad se concentra en las edades de 10 a 11 años. Los resultados de lateralidad muestran que la mayoría de los niños $(72,22 \%)$ eran diestros completos, $(27,78 \%)$ presentaban lateralidad indefinida y ningún niño presentaba lateralidad siniestra o cruzada. Conclusión: Es importante analizar la lateralidad de los niños porque, a partir de estas habilidades, pueden dominar sus movimientos y conocerse a sí mismos y al mundo que les rodea. Se sugiere realizar estudios similares, con muestras representativas, señalando datos que llamen la atención sobre una visión más amplia de la lateralidad en los niños, como prevención e intervención de probables distorsiones en el proceso de aprendizaje.
\end{abstract}

Palabras clave: Desarrollo motor; Lateralidad; Niños quilombola.

\title{
1. Introdução
}

O desenvolvimento motor infantil é caracterizado como um processo de mudança que depende da idade, postura e movimentos, estando também relacionado com o crescimento físico e o fortalecimento dos ossos, músculos e a capacidade de toque da criança (Xavier, 2018). Esse processo está intimamente relacionado a fatores ligados ao próprio indivíduo, ao ambiente e à tarefa executada em si (Marques et al., 2013). Assim, o avanço na aquisição de habilidades motoras caracteriza maior autonomia e competência para enfrentar a sociedade (Santos et al., 2016). Além do mais, para Silveira et al. (2014), o desenvolvimento motor pode ser influenciado por diversos fatores, sendo eles extrínsecos e/ou intrínsecos.

Os fatores intrínsecos (sistemas neuroendócrino e ósseo) e extrínsecos (influências ambientais e populacionais, nutrição, situação socioeconômica e atividade física) contribuem para que ocorra a variabilidade individual, pois podem ser manipulados, ou seja, acentuados, amenizados ou removidos, sendo esses fatores ainda, responsáveis pela diferenciação no ritmo de crescimento (Haywood \& Getchell, 2004, como citado em Pereira et al., 2010, p. 309)

Para Rosa Neto et al. (2010), o desenvolvimento motor das crianças é um método sequencial relacionado à idade real, causado pela interação entre a carga e tarefa, a biologia do indicador e as condições ambientais e é inerente às mudanças sociais, intelectuais e emocionais. Com a evolução da ciência, têm-se expandido os estudos sobre as disfunções do movimento, compreendidas como distúrbios de caráter multifatorial: peso ao nascer, status socioeconômico, nível de educação da mãe, fatores culturais, entre outros, podem estar interligados à desordem (Gondim et al., 2018).

Alguns autores enfatizam que esse processo está relacionado a fatores como a idade cronológica, e com o passar do tempo a criança vai adquirindo uma quantidade de habilidades motoras, progredindo assim de movimentos simples e desorganizados até a execução de habilidades motoras mais organizadas e elaboradas (Cotrim et al., 2011; Schardong, 2016). Essas habilidades são pertinentes à funcionalidade para a efetivação de tarefas, como o controle postural e o equilíbrio para executar atividades, saltar obstáculos, correr, além das variáveis sociais, comportamentais e cognitivas (Santos et al., 2016).

Para Haywood e Getchell (2016), esse processo é sequencial, contínuo e relacionado à idade cronológica, pela qual o ser humano adquire uma extensa quantidade de habilidades motoras, que evoluem por meio de movimentos simples e desordenados para a efetivação de movimentos organizados e complexos. O desenvolvimento é um processo longo, com início de movimentos reflexos evoluindo para movimentos primários e essenciais e, por último, para a fase dos movimentos elaborados (Gallahue \& Ozmun, 2005).

$\mathrm{Na}$ infância, é necessário considerar vários fatores para avaliar o que pode ou não ser considerado parte do desenvolvimento normal. Dentre esses fatores, os mais importantes estão no âmbito das diferenças culturais e dos aspectos econômicos e sociais, respeitando essas variantes, pode-se evitar a avaliação não compatível com os tipos de realidade existentes (Zattoni, 2011). 
Nesta fase da vida, ocorrem mudanças físicas rápidas e profundas em todo o corpo, inclusive no cérebro (Beltrame et al., 2019). Faz-se necessário considerar que o desempenho motor se encontra em progresso contínuo, iniciando de movimentos simples e realizados de forma individual para uma sucessão de combinações que serão usadas em atividades de lazer, esportivas e especialmente cotidianas e concomitantes aos processos da aprendizagem escolar e do amadurecimento das principais habilidades motoras (Gallahue \& Ozmun, 2005). É por meio das experiências vividas no cotidiano da criança que ela terá condições de desenvolver suas habilidades e ter controle sobre elas (Gomes, 2010).

A terceira infância corresponde à faixa etária dos 6 aos 12 anos incompletos (Papalia \& Feldman, 2013). Os autores Bee e Boyd (2011) a caracterizaram como a continuidade da meninice, marcada pelo desenvolvimento cerebral e crescimento dos 6 aos 8 anos e dos 10 aos 12 anos (Barros, 2018). Desse modo, cognitivamente a criança já se envolve em alguns desafios culturais e de inclusão de classe, compreende melhor a realidade do concreto e de si mesma, maximiza as estratégias cognitivas, o julgamento de valores e autoconceito aos 7 ou 8 anos e a separação de gênero aos 6 ou 7 anos (Barros, 2018). As mudanças do cognitivo e social não são feitas de forma rápida, na maioria das vezes a criança percebe algo aos 7 anos, mas só assimila aos 11 anos (Bee \& Boyd, 2011).

Com relação à simetria, muitas espécies, incluindo os seres humanos, são consideradas assimétricas, tanto em nível estrutural como funcional, sendo a assimetria funcional mais evidente no sistema nervoso, que diz respeito à mão e corresponde à preferência pelo uso do lado direito em vez do lado esquerdo do corpo, principalmente mão e pé, estando presente na maior parte da população (Willcutt, 2012). As relações do corpo que integram suas partes direita e esquerda constituem a lateralidade, que está vinculada ao processo de maturação dos centros sensórios motores de um dos hemisférios cerebrais (Le Bouclch, 2001)

A lateralidade é um conceito amplo que abrange distintos comportamentos dos seres vivos procedentes de um processamento no nível dos hemisférios cerebrais (Souza \& Teixeira, 2006). Entretanto, é sabido que a origem desse comportamento lateralizado ainda é discutível, porém há uma interação entre os fatores biológicos e ambientais (Fernandes et al., 2016).

Desde o estudo de Paul Broca sobre o hemisfério cerebral, houve muitas discursões sobre o domínio de um dos hemisférios cerebrais nas manifestações comportamentais e funcionais do ser humano (Lucena et al., 2010). Geralmente, a preferência manual é causada pela assimetria lateral, que se deve ao fato de a mão preferida ter maior capacidade de controle motor do que a mão não preferida. Para explicar a assimetria de desempenho lateral, é fornecido um conceito de que o gene tem especificações para a diferenciação e desenvolvimento dos hemisférios cerebrais, e é determinado que eles se tornarão os hemisférios dominantes em relação a certas funções (Souza et al., 2013).

Diante disso, o cuidado à saúde da criança deve visar especialmente o auxílio que coopera para o seu desenvolvimento, a partir do foco no ambiente e nas relações familiares, envolvendo sua rede social de apoio e uma conduta interdisciplinar (Gondim et al., 2018). Portanto, ações de educação básica são essenciais para manter as crianças nas melhores condições que levem à clara lateralização, de forma que respeite os fatores genéticos e ambientais, para que possam organizar suas atividades esportivas (Guimarães, 2014). Vale ressaltar que não é interessante forçar a criança a utilizar a postura oposta, aquela não utilizada frequentemente, mas sim que sejam estimuladas e/ou criadas situações para que ela utilize de forma espontânea seus membros (Souza et al., 2013).

O Ministério da Saúde brasileiro publica manuais técnicos sobre crescimento e desenvolvimento infantil com o intuito de fornecer informações cada vez mais assertivas (Brasil, 2002). A partir de levantamentos bibliográficos sobre essa abordagem, é possível verificar que há melhorias nas habilidades motoras de crianças que recebem estímulos (Marques et al., 2013).

Com passar do tempo o indivíduo adquire inúmeras habilidades motoras, o que enfatiza a importância de uma estimulação precoce na infância com a finalidade de lapidar ainda mais esse desenvolvimento motor, visto que se comprovam através da literatura progressos em crianças com essa estimulação motora (Guimarães, 2014). Nesse sentido, essa proposta de 
atuação apresenta-se importante na medida em que auxilia no entendimento do desenvolvimento motor de crianças quilombolas, a partir do pré e pós-teste que poderá identificar se alguma criança ainda não possui sua lateralidade totalmente definida.

Diante do exposto, ressalta-se que o estudo do desenvolvimento motor infantil traz as peculiaridades individuais e globais, seja com relação às questões ambientais, sociais, intelectuais e emocionais, seja relacionado ao quão exposta está a criança aos estímulos necessários para a construção de seu repertório motor. Portanto, associar os estímulos precoce de lateralidade com as individualidades de cada grupo social, como o quilombola, traz à literatura novas informações e padrões de desenvolvimento infantil. Sendo assim, o intuito da corrente pesquisa é analisar a lateralidade de crianças quilombolas e sua relação com o desenvolvimento motor infantil.

\section{Metodologia}

Trata-se de um estudo exploratório observacional, de caráter analítico, do tipo descritivo e transversal, com abordagem quantitativa e qualitativa (Estrela, 2018). A pesquisa foi realizada com uma amostra de 18 crianças, oriundas de uma área quilombola da comunidade Tiningu, localizada em região rural do oeste do estado, às margens do Rio Maicá (02 35' 49,54” S 54 29’ 34,85” W), a 47 km da área urbana de Santarém- Pará (INCRA/SR30, 2014). A comunidade possui uma área de 4269 ha, limitando-se ao norte com o Quilombo São José do Ituqui, ao sul com a comunidade indígena de Ipaupixuna e a oeste com o Quilombo de Murumuru (Brasil, 2013) (Figura 1).

Figura 1 - Mapa do quilombo Tiningu, Santarém -PA, Brasil. Área destacada em vermelho referente ao estudo.

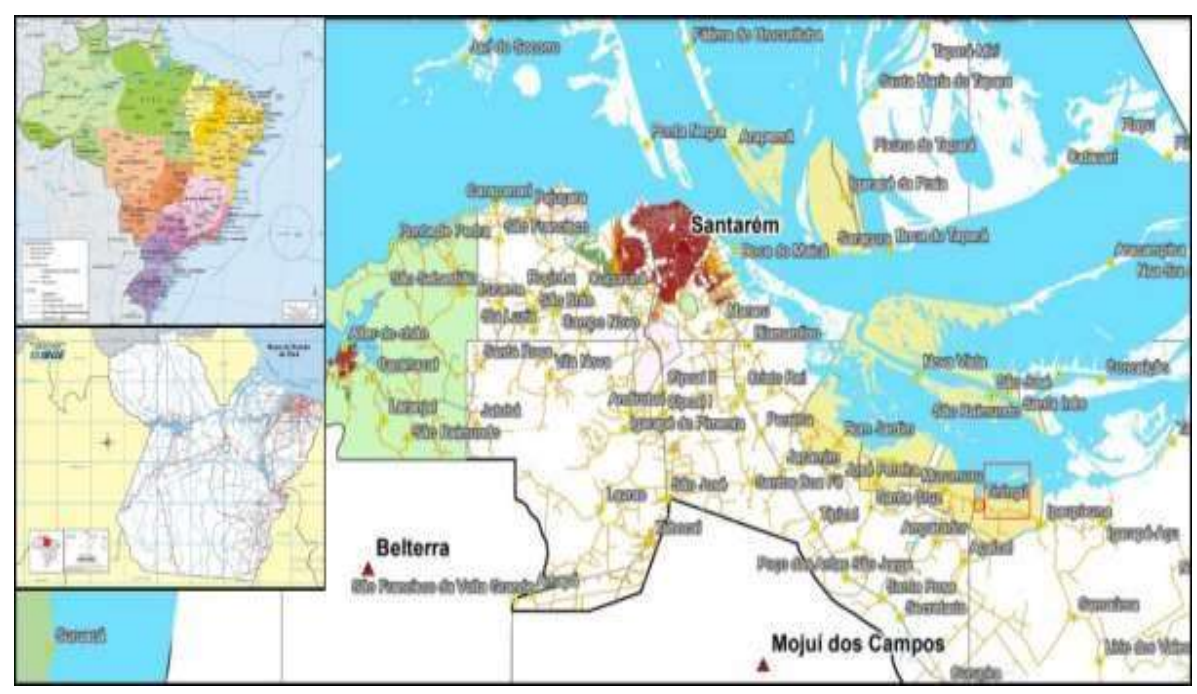

Fonte: Adaptado de Instituto Brasileiro de Geografia e Estatística (IBGE, 2013, n.d.), Projeto Saúde e Alegria (PSA, 2007) e Golobovante (2017).

Com base no instrumento de pesquisa, foram analisadas 18 crianças com idade de 7 a 11 anos (12 anos incompletos), sendo 11 meninas e 7 meninos, que estavam na terceira infância de uma amostragem que incluía (N) 39 crianças. Após uma semana de organização e transcrição dos dados, foi então disponibilizada pelo ASC uma triagem da amostragem.

Foram incluídos no grupo amostral meninos e meninas de 7 a 11 anos que vivem em áreas reconhecidas pelo Instituto Nacional de Colonização e Reforma Agrária (INCRA) como remanescentes de quilombo cujos pais/responsáveis permitiram a participação.

Excluíram-se do estudo as crianças cujos pais e responsáveis não concordam com o Termo de Consentimento Livre e Esclarecido (TCLE) (Anexo B); crianças que não assinaram o Termo de Consentimento Livre e Esclarecido (TALE) (Anexo C) 
não participarão do estudo; e as crianças que tinham problemas mentais e/o outras atividades temporárias como, por exemplo, desenho, não estão envolvidos.

Utilizou-se como instrumento de coleta de dados a Escala de Desenvolvimento Motor Infantil (EDM) de Rosa Neto (2002). Essa ferramenta avalia as seguintes áreas do desenvolvimento: motricidade fina e geral; equilíbrio; esquema corporal; organização espacial e temporal; e lateralidade.

Assim, foram analisadas as Idades Motoras (IM) e o Quociente Motor (QMG). As baterias dos testes consistem em 10 tarefas motoras para cada uma das seis áreas do desenvolvimento, distribuídas entre 2 e 11 anos, organizadas de acordo com grau de complexidade, progressivamente, sendo atribuído para cada tarefa, em caso de êxito, um valor correspondente à idade motora, expressa em meses. Em cada bateria, o teste é interrompido caso a criança não alcance o êxito. Ao final da aplicação, dependendo do desempenho, é atribuída uma idade motora, em cada uma das áreas referidas (Rosa Neto, 2002).

Dessa forma, para comparar os resultados, inicialmente é determinada a Idade Cronológica (IC) e transformada a idade do avaliado em meses. A IM é determinada a partir dos testes e também transformada em meses. Computa-se então a idade motora geral (IMG) através do seguinte cálculo: IM1+IM2+IM3+IM4+IM5+IM6/6 para se achar a medida da idade motora. Comparando-se a IC com a IMG, pode-se determinar o avanço ou o atraso da criança através da obtenção do Quociente Motor Geral (QMG), que implica na divisão da IMG e pela IC multiplicada por 100.

Os resultados do QMG serão classificados através das seguintes pontuações: acima de 130 (muito superior); 120-129 (superior); 110-119 (normal alto); de 90-109 (normal médio); 80-89 (normal baixo); $70-79$ (inferior); 69 ou menos (muito inferior) (Rosa Neto, 2002).

Os dados sociodemográficos foram analisados mediante estatísticas descritivas, usando os programas Microsoft Office Excel 2010 e BioEstat versão 5.0. A análise descritiva foi realizada por meio de médias, frequência máxima, mínima e desvio padrão.

Os dados da EDM foram tabulados e analisados de acordo com a proposta de Rosa Neto (2002), que permitiu a análise das áreas motoras que compreendem o desenvolvimento motor, evidenciando os pontos fortes e fracos das crianças desta pesquisa. Esses dados passaram por análise estatística descritiva simples no Microsoft Office Excele 2010 e BioEstat versão 5.0.

Todos os resultados do presente estudo fizeram parte dos objetivos específicos de uma pesquisa maior da Universidade Federal do Oeste do Pará, aprovada pelo Comitê de Ética e Pesquisa (CEP) da Universidade do Estado do Pará (UEPA), Campus XII - Santarém, Pará, com número de parecer 3.303.675, assinado pelo coordenador desse Comitê em 06 de maio de 2019, sendo liberado o início dos procedimentos legais que conduziram este estudo.

Logo, este estudo foi desenvolvido seguindo a resoluções n 466/2012 e 510/2016, do Conselho Nacional de Saúde, conforme Norma Operacional nº 001/2013, sobre pesquisa envolvendo seres humanos, do Conselho Nacional de Saúde (CNS), sendo respeitados os princípios de autonomia, beneficência, não maledicência, justiça e equidade.

Também foi solicitada a autorização para a realização da pesquisa junto à Federação das Organizações Quilombolas de Santarém - Pará (FOQS), conforme a Instrução Normativa do PPGSAQ da UFOPA, tendo em vista que a comunidade de Tiningu é uma das 12 comunidades representadas pela FOQS.

\section{Resultados}

A amostra é composta por 18 crianças dos gêneros feminino ( $n=11)$, a maioria, e masculino ( $n=7)$, com faixa etária de 7 a 11 anos. Notou-se que a faixa etária em maior quantidade se concentra nas idades de 10 a 11 anos (56\%), composta em grande parte por meninas. Já os valores das idades dos meninos estão distribuídos entre 7 e 11 anos, com uma pequena concentração nos intervalos de 7 a 8 anos. 
A Tabela 1 apresenta as medidas estatísticas das idades cronológicas (IC) em meses das crianças, agrupadas de forma geral e por gêneros. Ela mostra uma média das IC de 111,67 meses, sendo que a médias de IC em meses das meninas (116,73) é maior que a dos meninos $(105,14)$, em que os valores máximos demostraram ser de 132 meses em todas as categorias da tabela, e os valores mínimos foram desiguais nas IC das meninas que apresentaram a maior idade, correspondente a 87 meses.

Tabela 1 - Medidas estatísticas da IC em meses das crianças.

\begin{tabular}{ccccccccc}
\hline Características & Média & $\begin{array}{c}\text { Desvio } \\
\text { Padrão }\end{array}$ & Moda & Mediana & $\begin{array}{c}\text { Valor } \\
\text { Máximo }\end{array}$ & $\begin{array}{c}\text { Valor } \\
\text { Mínimo }\end{array}$ & $\begin{array}{c}\text { Tamanho } \\
\text { (n) }\end{array}$ \\
\hline Amostra (n) & 111,67 & 19,46 & 132 & 120,50 & 132 & 84 & 18 & 11 \\
Meninas & 116,73 & 17,17 & 132 & 124 & 132 & 87 & 11 \\
Meninos & 105,14 & 20,23 & 132 & 98 & 132 & 84 & 7 \\
\hline
\end{tabular}

Fonte: Autores (2019).

A Tabela 1 mostra também a medida das posições dessas IC, destacando a diferença dos valores da média e da mediana, em que os valores da mediana dos meninos correspondem a 98 meses e das meninas correspondem a 124 meses, enquanto a média aritmética é de 105,14 meses para os meninos e 116,73 meses para as meninas. Porém, a idade que se repetiu mais vezes nas três amostras de idades foi a de 132 meses, mostrando a superioridade das crianças com essa idade na amostra total e por divisão de gênero.

Abaixo, o Gráfico 1 apresenta o box-plot para as IC (em meses) por gênero comparadas com a IC geral. Aparentemente, observa-se que existem diferenças entre as três médias, com destaque para a média das meninas, que é maior que as demais.

Gráfico 1 - Distribuições das crianças por IC em meses.

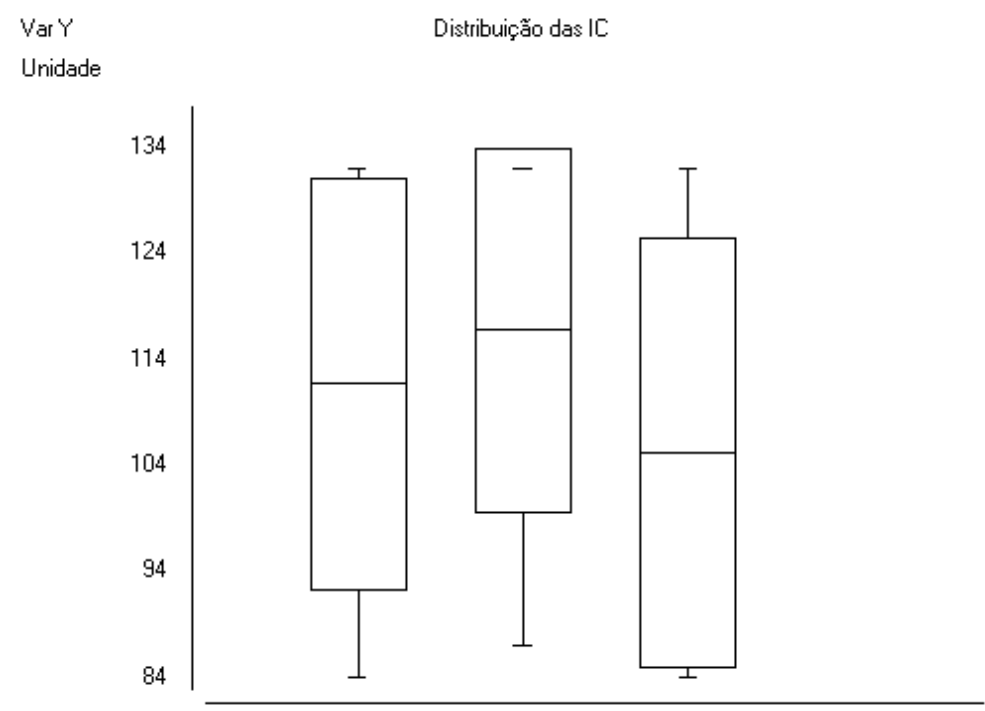

Nota. Eixo x - IC geral, IC meninas, IC meninos, respectivamente; Eixo Y - IC em meses. Fonte: Autores (2019).

Os resultados de lateralidade das crianças avaliadas no presente estudo estão alocados na Tabela 2. Nela, constata-se que a maioria das crianças, 72,22\% (n=13), são destras completas, ou seja, apresentam lateralidade direita de mãos, olhos e pés, e $27,78 \%(n=5)$ apresentam lateralidade indefinida (arremessam com a mão e o pé esquerdo, mas foca o olhar pelo orifício com o olho direito, por exemplo). E nenhuma criança apresentou lateralidade sinistra ou cruzada. 
Tabela 2 - Frequência percentual da avaliação de lateralidade.

\begin{tabular}{ccc}
\hline LATERALIDADE & $\mathbf{N}=$ & $\boldsymbol{\%}$ \\
\hline Destro Completo & 13 & 72,22 \\
Sinistro & 0 & 0 \\
Cruzada & 0 & 0 \\
Indefinida & 5 & $27,78 \%$ \\
\hline TOTAL & 18 & 100 \\
\hline
\end{tabular}

Fonte: Autores (2019).

Nos dados da tabela acima, percebe-se também a ausência nos achados desse teste de lateralidade de crianças com classificação como sinistra, com dominância do lado esquerdo (mãos, pés e olhos).

\section{Discussão}

Traçando um comparativo com outras pesquisas que abordam a mesma temática, verificou-se a importância da lateralidade no desenvolvimento infantil. Uma pesquisa envolvendo 20 crianças indicou que a idade motora se equiparou à idade cronológica, mostrando que estimulação motora é fundamental na infância, pois irá garantir que esse período traga condições propícias para o desenvolvimento ao longo da vida adulta (Forchezatto \& Fachineto, 2009). Isso corrobora as perspectivas de Meur e Staes (1991) a respeito do desenvolvimento motor e lateralidade, em que a lateralidade é extremamente importante para o desenvolvimento infantil por influenciar diretamente nas noções que as crianças têm de si mesmas, na formação de seu esquema corporal e na percepção da simetria do corpo. Para o autor, o desenvolvimento dessa habilidade contribui para determinar a estruturação espacial, pois ao perceber o eixo de seu corpo, a criança percebe também seu meio ambiente em relação a esse eixo (Meur \& Staes, 1991).

Na corrente pesquisa, pôde-se constatar que a maioria das crianças $(72,22 \%, n=13)$ são destras completas, ou seja, apresentam lateralidade direita de mãos, olhos e pés. Já no estudo de Lucena et al. (2010), realizado com 400 crianças na faixa etária de 6 a 10 anos, foram observados 23 tipos de lateralidade mão-olho-pé, isto é, as dominâncias manual, ocular e dos membros inferiores conjuntas, havendo predomínio para a utilização do lado direito nas três situações. É interessante notar, também, o maior percentual $(48,75 \%)$ de destralidade completa (dominância manual, ocular e dos membros inferiores direita). Os resultados apontam que houve associação significativa $(\mathrm{p}<0,05)$ entre sinistralidade completa e déficit de organização. O uso exclusivo da mão direita na realização de tarefas também se mostrou compatível com os dados da população geral relatados por Habib (2000). Existe a probabilidade de os destros representarem mais de $90 \%$ dos indivíduos no mundo, qualquer que seja seu meio cultural, sendo que $70 \%$ parecem ser destros manuais completos, os quais efetuam todas as tarefas exclusivamente com a mão direita (Habib, 2000).

No que diz respeito à lateralidade indefinida, $27,78 \%$ das crianças $(\mathrm{n}=5)$ apresentaram esse padrão, no qual arremessam com a mão e o pé esquerdo, mas focam o olhar pelo orifício com o olho direito, por exemplo. Além disso, nenhuma criança apresentou lateralidade sinistra ou cruzada. Por outro lado, na pesquisa de Lucena et al. (2010), o número de casos de discrepância entre as três dominâncias, presente em 48,8\% dos casos investigados pelos autores, traduziu lateralidades cruzadas com predominância direita (D), sendo DED, DDE ou EDD, ou com predominância esquerda (E), sendo EDE, EED ou DEE, ou lateralidades mal definidas, observadas nos demais casos discrepantes entre mão, olho e pé.

Silva et al. (2017) averiguaram a lateralidade das crianças praticantes de exercícios físicos, na faixa etária de 8 a 11 anos, sendo encontrado: a maioria indefinidas $(53,34 \%)$, destras $(26,66 \%)$ e sinistras $(20 \%)$. Entre as crianças não praticantes encontraram-se: indefinidas $(46,68 \%)$, cruzadas $(33,33 \%)$, destras $(13,33 \%)$ e sinistras $(6,66 \%)$. Tais resultados corroboram a 
ideia de Santos et al. (2016) de que é o ambiente que proporciona estímulos para ajudar no desenvolvimento dos componentes da motricidade e tem capacidade de auxiliar no domínio do controle motor e maximizar o desenvolvimento motor.

A lateralidade atua como variável do desempenho motor e psicológico, além de ser um dos aspectos mais relevantes em relação ao desenvolvimento das capacidades de aprendizagem e pode ser definida como a capacidade de integração sensóriomotora dos dois lados do corpo, sendo então, de um modo geral a preferência da utilização de um desses lados (Rosa Neto, 2002).

Ao comparar o desenvolvimento motor em crianças praticantes e não praticantes de exercícios físicos, Silva et al. (2017) puderam constatar que as crianças praticantes de exercícios apresentaram a maior média de quociente motor geral $(109,4)$ apresentando-se com o desenvolvimento motor superior que os não praticantes, de acordo com a Escala de Desenvolvimento Motor. Essas habilidades obtidas pelas crianças são pertinentes à funcionalidade para a efetivação de tarefas, como o controle postural e o equilíbrio para executar atividades, saltar obstáculos, correr, além das variáveis sociais, comportamentais e cognitivas (Santos et al., 2016).

O desenvolvimento motor infantil é um método sequencial relacionado à idade cronológica, provocado pela interação entre os encargos da tarefa, a biologia individual e as condições ambientais, e é inerente às mudanças sociais, intelectuais e emocionais (Rosa Neto et al., 2010). É importante que a criança experimente os dois lados, sem interferências, já que as potencialidades para destros e sinistros é a mesma, desde que tenha desenvolvido de forma natural sua lateralidade (Brandão, 1984). No entender de Oliveira (2010), as perturbações na lateralização podem resultar em efeitos negativos na aprendizagem. Além disso, a criança que não desenvolveu de forma natural sua lateralidade pode apresentar dificuldades para aprender a direção gráfica e escrita especular, bem como problemas na discriminação visual e estruturação espaço-temporal e com os conceitos esquerda e direita, o que pode comprometer o aprendizado da leitura e da escrita. Portanto, uma lateralidade bem definida auxilia a criança a assimilar conceitos espaciais e, assim, diferenciar os lados de seu eixo corporal (Oliveira, 2010; Santi Maria, 2012).

\section{Conclusão}

A partir dos resultados do presente estudo, foi possível verificar o nível de lateralidade das crianças residentes na área quilombola da comunidade Tiningu. Com isso, fica claro que o ambiente em que a criança está inserida proporciona estímulos para ajudar no seu desenvolvimento motor.

É importante analisar a lateralidade das crianças e o quanto pode influenciar em suas vidas, pois a partir dessa habilidade elas podem elaborar melhor e dominar seus movimentos, além de ter o conhecimento de si mesmas e do mundo ao seu redor. É imprescindível, também, deixar que as crianças desenvolvam de forma natural a sua lateralidade.

Constatamos que a estimulação motora é fundamental na terceira infância, pois irá garantir que esse período traga condições propícias para o seu desenvolvimento ao longo da vida, além de melhorar esse aspecto das crianças que já possuem uma lateralidade definida, sejam elas destras completas, sinistras completas ou apresentem lateralidade cruzada, e faça com que as crianças que possuem lateralidade indefinida consigam defini-la através de atividades funcionais estimuladas para que experimentem os dois lados, sem interferências.

Espera-se, com a corrente pesquisa, oferecer novas percepções a respeito da temática em questão. Sugere-se a realização de estudos similares, com amostras representativas, apontando dados que chamem a atenção para uma visão mais ampla da lateralidade funcional em crianças, como meio de prevenir e intervir em prováveis distorções no processo de aprendizagem.

\section{Referências}

Barros, M. I. A. D. (2018). Desemparedamento da infância: a escola como lugar de encontro com a natureza. Programa Criança e Natureza; Alana.

Beltrame, R. L., Gesser, M., \& Souza, S. V. D. (2019). Diálogos sobre medicalização da infância e educação: uma revisão de literatura. Psicologia em Estudo, 24. https://doi.org/10.4025/psicolestud.v24i0.42566 
Boyd, D., \& Bee, H. (2011). A criança em crescimento. Artmed.

Brandão, J. S. (1984). Desenvolvimento psicomotor da mão. Enelivros.

Brasil. (2002). Saúde da criança: acompanhamento do crescimento e desenvolvimento infantil. Ministério da Saúde.

Brasil. (2013). Relatório da pesquisa quantitativa em nove comunidades quilombolas de Santarém, Pará. Projeto Brasil Local Etnodesenvolvimento e Economia Solidária.

Cotrim, J. R., Lemos, A. G., Néri Júnior, J. E., \& Barela, J. A. (2011). Desenvolvimento de habilidades motoras fundamentais em crianças com diferentes contextos escolares. Revista da Educação Física/UEM, 22, 523-533.

Estrela, C. (2018). Metodologia científica: ciência, ensino, pesquisa. Artes Médicas.

Forchezatto, R., \& Fachineto, S. (2009). Lateralidade e equilíbrio em crianças de $1^{\text {a }}$ a $3^{\text {a }}$ série do município de Descanso/SC-diagnóstico e proposta de intervenção. Cinergis, 10(1).

Fernandes, J. O, Willrich, A., \& Azevedo, C. C. F. (2016). Desenvolvimento motor na infância: influência dos fatores de risco e programas de intervenção. Revista Neurociências, 17(1), 51-56.

Gallahue, D.L., \& Ozmun, J. C. (2005). Compreendendo o desenvolvimento motor: bebês, crianças, adolescentes e adultos (3a ed.). Phorte.

Golobovante, F. (2017). Limites Municipais - IBGE Massa D'água e Vias de Acesso - Imazon Localidades e Áreas Protegidas - CIAM/SEMMA [Mapa]. IBAMA.

Gomes, L. B. (2010). Atletismo como Esporte Base no Desenvolvimento Motor. Clube de Autores.

Gondim, G. D. M. C., Sá, F. E. D., \& Cardoso, K. V. V. (2018). Caracterização do desenvolvimento motor de crianças em vulnerabilidade social de uma creche filantrópica [Trabalho de Conclusão de Curso]. Repositório Institucional UFC. http://repositorio.ufc.br/handle/riufc/39640

Guimarães, A. S. (2014). Motricidade global e lateralidade de crianças de 7 a 11 anos praticantes de natação [Trabalho de Conclusão de Curso]. RUNA Repositório Universitário da Ânima. https://repositorio.animaeducacao.com.br/handle/ANIMA/12933

Habib, M. (2000). Bases neurológicas dos comportamentos. Climepsi.

Haywood, K. M., \& Getchell, N. (2004). Desenvolvimento motor ao longo da vida (3a ed.). Artmed.

Haywood, K. M., \& Getchell, N. (2016). Desenvolvimento Motor ao Longo da Vida (6a ed.). Artmed.

Instituto Brasileiro de Geografia e Estatística. (2013). Base Cartográfica Vetorial Contínua do Brasil, ao Milionésimo - bCIMd: versão 2.1 para ArcGis Desktop/Arcview [Mapa]. IBGE.

Instituto Brasileiro de Geografia e Estatística. (n.d.). Mapa político do Brasil [Mapa]. IBGE. https://www.guiageografico.com/mapas/mapa-brasil.htm

Instituto Nacional de Colonização e Reforma Agrária. (2014). http://www.incra.gov.br/index.php/para--santarem-sr-30. Acessado em 12 de maio de 2014.

Le Boulch, G. (2001). Approche systémique de la proximité: définitions et discussion. 3ème Journées de la Proximité, Paris.

Lucena, N. M. G. D., Soares, D. D. A., Soares, L. M. D. M. M., Aragão, P. O. R. D., \& Ravagni, E. (2010). Lateralidade manual, ocular e dos membros inferiores e sua relação com déficit de organização espacial em escolares. Estudos de Psicologia, 27, 03-11.

Marques, T. S., Vilela, J. G. R., Figueiredo, B. D., \& Figueiredo, A. P. (2013). Desenvolvimento motor: padrões motores fundamentais. Revista Digital EFDeportes, 18, 186

Meur, A., \& Staes, L. (1991). Psicomotricidade: educação e reeducação, níveis: maternal e infantil. Manole.

Oliveira, G. de C. (2010). Avaliação Psicomotora à luz da psicologia e da psicopedagogia. Vozes.

Pereira, P. C., Manzatto, L., \& Marco, A. de (2010). Análise do crescimento e desenvolvimento motor de escolares de $1^{\text {a }}$ a $4^{\mathrm{a}}$ série do município de HolambraSão Paulo. HU Revista, 36(4)

Projeto Saúde e Alegria. (2007). Comunidades Remanescentes de Quilombos Santarém [Mapa]. PSA,

Rosa Neto, F. (2002). Manual de avaliação motora (Vol. 1). Artmed.

Rosa Neto, F., Santos, A. P. M. D., Xavier, R. F. C., \& Amaro, K. N. (2010). A Importância da avaliação motora em escolares: análise da confiabilidade da Escala de Desenvolvimento Motor. Revista Brasileira de Cineantropometria \& Desempenho Humano, 12, $422-427$.

Santi Maria, T. L. C. (2012). Desenvolvimento psicomotor de alunos na Educação Infantil [Dissertação de mestrado]. Biblioteca Digital Brasileira de Teses e Dissertações. https://bdtd.ibict.br/vufind/Record/CAMP_21f318a0d5a91be1e84962674124484c

Santos, A. P. M., Villaverde, L. N., Costa, A. N. F., Santos, M. de O., Gregório, E. C., Andreis, L. M., \& Neto, F. R. (2016). Aspectos biopsicossociais em escolares com atraso no desenvolvimento motor: um estudo longitudinal. Journal of Human Growth and Development, 26(1), 112-118. https://dx.doi.org/10.7322/jhgd.113787 
Research, Society and Development, v. 10, n. 15, e68101522750, 2021

(CC BY 4.0) | ISSN 2525-3409 | DOI: http://dx.doi.org/10.33448/rsd-v10i15.22750

Schardong, F. R. (2016). O estudo das ações motoras nas perspectivas desenvolvimentista ecológica e histórico-cultural [Dissertação de mestrado]. Repositório UNESC. http://repositorio.unesc.net/handle/1/3995

Silva, T. R., Contenças, T. S., \& Marques, A. (2017). Avaliação do desenvolvimento motor em crianças praticantes e não praticantes de exercícios físicos. Revista Brasileira de Fisiologia do Exercício, 16(4), 208-214.

Silveira, R. A. da, Cardoso, F. L., \& de Souza, C. A. (2014). Avaliação do desenvolvimento motor de escolares com três baterias motoras: EDM, MABC-2 e TGMD-2. Cinergis, 15(3).

Souza, L. P. T. D., Figueiredo, B. M. D., \& Figueiredo, A. P. (2013). A relação entre desenvolvimento motor, lateralidade e iniciação esportiva. EFDeportes.com, 18(184). https://www.efdeportes.com/efd184/lateralidade-e-iniciacao-esportiva.htm

Souza, R. M. D., \& Teixeira, L. A. (2011). Sobre a relação entre filogenia e ontogenia no desenvolvimento da lateralidade na infância. Psicologia: Reflexão e Crítica, 24, 62-70.

Willcutt, E. G. (2012). The prevalence of DSM-IV attention-deficit/hyperactivity disorder: a meta-analytic review. Neurotherapeutics, 9(3), 490-499.

Xavier, J. (2018). A importância do desenvolvimento motor na primeira infância. http://www.iff.fiocruz.br/index.php/8-noticias/457-a-importancia-dodesenvolvimento-motor-na-primeira-infancia

Zattoni, R. S. (2011). A autoestima em crianças da terceira infância e sua relação com o elogio no contexto educacional [Apresentação de trabalho]. X congresso nacional de educação-EDUCERE, \& I seminário internacional de representações sociais, subjetividade e educação-SIRSSE, Curitiba. 